\title{
Chemical Synthesis of $\beta-\mathrm{Ga}_{2} \mathrm{O}_{3}$ Microrods on Silicon and Its Dependence on the Gallium Nitrate Concentration
}

Guislain Hector, ${ }^{1}$ Estelle Appert, ${ }^{1}$ Eirini Sarigiannidou, ${ }^{1}$ Eléa Matheret, ${ }^{1}$ Hervé Roussel, ${ }^{1}$ Odette Chaix-Pluchery, ${ }^{1}$ and Vincent Consonni. ${ }^{1 *}$

${ }^{1}$ Univ. Grenoble Alpes, CNRS, Grenoble INP, LMGP, F-38000 Grenoble, France

\section{*Corresponding Author:}

Vincent Consonni

LMGP, Grenoble INP - Minatec, 3 parvis Louis Néel - CS 50257 - 38016 Grenoble cedex 1

Tel : (+33) 456529358

vincent.consonni@grenoble-inp.fr

\section{SUPPORTING INFORMATION}

\begin{tabular}{|c|c|}
\hline Chemical reaction & $\begin{array}{c}\text { Log } \\
(\mathrm{K})\end{array}$ \\
\hline $\mathrm{Ga}^{3+}+\mathrm{OH}^{-} \Leftrightarrow \mathrm{GaOH}^{2+}$ & 11.1 \\
$\mathrm{Ga}^{3+}+2 \mathrm{OH}^{-} \Leftrightarrow \mathrm{Ga}(\mathrm{OH})_{2}^{+}$ & 9.16 \\
$\mathrm{Ga}^{3+}+4 \mathrm{OH}^{-} \Leftrightarrow \mathrm{Ga}(\mathrm{OH})^{-}$ & 2.98 \\
\hline
\end{tabular}

Table S1. Possible chemical reactions in aqueous solution and related equilibrium constants $\mathrm{K}$ at $25^{\circ} \mathrm{C}$, considering the $\mathrm{Ga}-\mathrm{OH}$ chemical system.

\begin{tabular}{|l|r|}
\hline \multicolumn{1}{|c|}{ Solubility } & \multicolumn{1}{c|}{$\begin{array}{c}\text { Log } \\
(\mathrm{Ks})\end{array}$} \\
\hline $\mathrm{Ga}(\mathrm{OH})_{3}(\mathrm{am})$ & 4.99 \\
$\mathrm{GaOOH}(\mathrm{s})$ & 2.89 \\
\hline
\end{tabular}

Table S2. Solubility constants $\mathrm{K}_{\mathrm{s}}$ at $25^{\circ} \mathrm{C}$ for the solid phases of gallium-based compounds 


\begin{tabular}{|c|c|c|c|c|c|c|}
\hline Compound & $2 \theta\left(^{\circ}\right)$ & (hkl) plane & $\mathrm{I}_{0, \mathrm{hk} 1}$ & $\mathrm{I}_{\mathrm{hkl}}$ & $\mathrm{C}_{\mathrm{hkl}}$ & $\sigma$ \\
\hline $\begin{array}{c}\alpha-\mathrm{GaOOH} \\
\mathrm{ICDD}(04-010-9861)\end{array}$ & $\begin{array}{l}18.063 \\
21.416 \\
26.621 \\
33.655 \\
35.296 \\
36.596 \\
37.210 \\
39.366 \\
40.466 \\
41.743\end{array}$ & $\begin{array}{l}(200) \\
(101) \\
(201) \\
(301) \\
(210) \\
(400) \\
(111) \\
(002) \\
(102) \\
(401)\end{array}$ & $\begin{array}{r}10 \\
100 \\
12 \\
40 \\
28 \\
16 \\
74 \\
4 \\
4 \\
14\end{array}$ & $\begin{array}{r}2155 \\
37411 \\
1225 \\
3114 \\
1247 \\
2798 \\
1684 \\
558 \\
361 \\
1014\end{array}$ & $\begin{array}{l}1.68 \\
2.86 \\
0.76 \\
0.60 \\
0.35 \\
1.37 \\
0.17 \\
0.97 \\
0.67 \\
0.57\end{array}$ & 0.74 \\
\hline $\begin{array}{c}\beta-\mathrm{Ga}_{2} \mathrm{O}_{3} \\
\mathrm{ICDD}(00-041-1103)\end{array}$ & $\begin{array}{l}15.73 \\
18.96 \\
30.10 \\
31.68 \\
33.46 \\
35.10 \\
37.38 \\
38.35 \\
45.80 \\
64.10\end{array}$ & $\begin{array}{c}(001) \\
(-201) \\
(400) \\
(002)(-202) \\
(-111) \\
(111) \\
(401) \\
(-311) \\
(600)(-312) \\
(-204)\end{array}$ & $\begin{array}{r}3 \\
16 \\
54 \\
100 \\
17 \\
68 \\
19 \\
38 \\
20 \\
16\end{array}$ & $\begin{array}{r}294 \\
323 \\
1026 \\
6910 \\
104 \\
341 \\
200 \\
405 \\
144 \\
342\end{array}$ & $\begin{array}{l}3.67 \\
0.76 \\
0.71 \\
2.59 \\
0.23 \\
0.19 \\
0.39 \\
0.40 \\
0.27 \\
0.80\end{array}$ & 1.11 \\
\hline
\end{tabular}

Table S3. Texture coefficients $C_{h k l}$ and degree of preferred orientation $\sigma$ for the main diffraction peaks, along with their intensities for $\alpha-\mathrm{GaOOH}$ and $\beta-\mathrm{Ga}_{2} \mathrm{O}_{3}$ microrods.

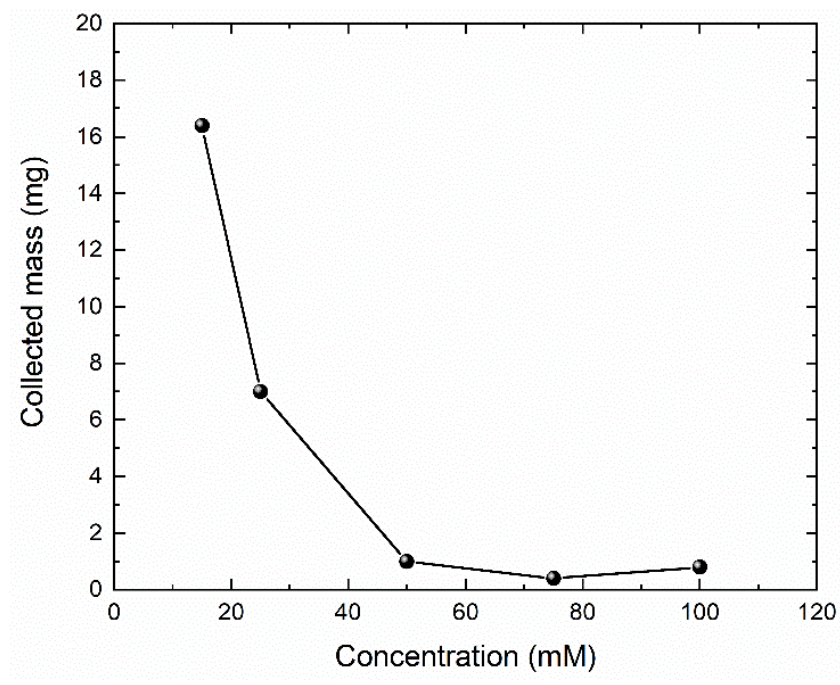


Figure S1. Collected mass plot of homogeneous precipitate as a function of the $\mathrm{Ga}\left(\mathrm{NO}_{3}\right)_{3}$ concentration. 